\title{
Radiotherapy of brain metastases from small-cell lung cancer: standards and controversies
}

\author{
Lucyna Kepka \\ Department of Radiation Oncology Military Institute of Medicine, Warsaw 04-141, Poland.
}

Correspondence to: Dr. Lucyna Kepka. Department of Radiation Oncology Military Institute of Medicine, Warsaw 04-141, Poland.E-mail: Ikepka@wim.mil.pl

How to cite this article: Kepka L. Radiotherapy of brain metastases from small-cell lung cancer: standards and controversies. J Cancer Metastasis Treat 2019;5:53. http://dx.doi.org/10.20517/2394-4722.2018.114

Received: 31 Dec 2018 First Decision: 15 Apr 2019 Revised: 16 May 2019 Accepted: 16 May 2019 Published: 11 Jul 2019

Science Editor: Ira-Ida Skvortsova Copy Editor: Cai-Hong Wang Production Editor: Jing Yu

\begin{abstract}
Small-cell lung cancer (SCLC) has a high propensity to metastasize into the brain. Radiotherapy plays a major role in the treatment of brain metastases (BM) from SCLC. Whole-brain radiotherapy (WBRT) is the standard treatment of BM from SCLC. However, the neurocognitive toxicity and modest efficacy of this approach have led to the increased use of stereotactic radiosurgery. We have no strong evidence for the use of different forms of radiation (WBRT vs. radiosurgery) in SCLC, because BM from this primary tumor were excluded from clinical trials. In this review, the use of radiation in form of WBRT or radiosurgery is discussed in distinct clinical indications: as a primary treatment and at relapse; without prior use of prophylactic cranial irradiation ( $\mathrm{PCl}$ ); and after $\mathrm{PCl}$. Combinations of radiotherapy with chemotherapy are discussed as BM in SCLC occur rarely as a sole event.
\end{abstract}

Keywords: Small-cell lung cancer, brain metastases, whole-brain radiotherapy, radiosurgery

\section{INTRODUCTION}

Small-cell lung cancer (SCLC) has a higher propensity than other solid tumors to spread to the brain. As many as 40\%-50\% of SCLC patients will develop brain metastases (BM) during the course of their disease ${ }^{[1]}$. Apart from a higher risk of occurrence than in other cancers, BM from SCLC have distinct clinical characteristics that differ from BM from all other solid tumors. These differences are reflected in radioand chemo-sensitivity and high aggressiveness with rapid propagation in the brain. The latter property makes BM from SCLC hardly suitable for local treatment such as surgery or radiosurgery. Unlike BM from

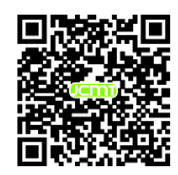


other cancers whole brain radiotherapy (WBRT) remains a standard treatment even if BM are suitable for radiosurgery. The well recognized radio- and chemo-sensitivity of SCLC are also present in the case of BM. Despite their radio- and chemo-responsiveness, the prognosis of BM from SCLC remains very poor with median survival after WBRT of 3.0-4.7 months in both retrospective and prospective studies ${ }^{[2-5]}$. The high risk of developing $\mathrm{BM}$ with a dismal disease course once BM have occurred and their high radiosensitivity have led to many trials assessing the value of prophylactic cranial irradiation (PCI). To date, SCLC is the only solid tumor in which the prolongation of survival with the use of PCI has been demonstrated for localized stage (LS) SCLC in a meta-analysis ${ }^{[6]}$. A total dose of 25 Gy in 10 fractions was established in a randomized trial as a standard dose of PCI for responders to initial therapy of LS SCLC ${ }^{[7]}$. In addition, for extensive stage (ES) SCLC, there were some indications that the use of PCI is of value for prolongation of survival ${ }^{[6]}$ and this was demonstrated in one randomized trial ${ }^{[8]}$.

The present review summarizes the problems related to radiotherapy of BM from SCLC with an emphasis on the distinctiveness of this approach in relation to management of BM from solid tumors. Different approaches in specific indications for treatment of BM from SCLC are discussed.

\section{PROGNOSTIC FACTORS}

Patients with BM from SCLC are usually excluded from trials on management of BM due to the different biological behavior of the primary tumor. The question arises if existing prognostic scores for overall survival in patients with BM are also relevant for BM from SCLC. The pivotal RTOG prognostic score was published after carrying out a recursive partitioning analysis (RPA) of pretreatment characteristics of patients in the randomized trials on BM. The prognosis of BM patients was related to the presence of three prognostic factors: performance status, presence of extracranial disease and age. Class 1 with the best prognosis included patients younger than 65 years, with control of extracranial disease, and with performance status $>60$ in Karnofsky performance status (KPS) score. Class 3 with the poorest prognosis included patients with KPS $<70$ regardless of the presence of all other factors. Class 2 with intermediate prognosis included patients who did not meet the criteria of classes 1 or $3^{[9]}$. These three prognostic factors are still the basis of all contemporary, more refined prognostic scores, even if new factors are included. However, in the original RTOG database, only $4 \%$ of patients had SCLC histology ${ }^{[9]}$. Retrospective studies have confirmed the validity of the prognostic stratification of BM from SCLC according to the RTOG RPA class criteria. In 132 SCLC patients who received WBRT, the median survival for classes 1,2 , and 3 was $2,4.5$, and 2 months, respectively $(P=0.003)$. However, there were only eight $(4 \%)$ patients in class $1^{[2]}$. In 154 patients with $\mathrm{BM}$ of whom $98 \%$ received WBRT as part of their treatment, the median survival for classes 1,2 , and 3 was 8.6, 4.2, and 2.3 months, respectively $(P=0.002)$. Only $5 \%$ of patients from this group met the criteria of RPA class $1^{[2]}$. The rarity of RPA class 1 among patients treated for BM from SCLC is related to the aggressiveness of the primary tumor. Brain-only metastases from SCLC with cured primary are an extremely rare event, as demonstrated by EORTC phase II trial, in which the accrual of patients with brain-only metastases was so slow that the study was stopped before the required number of patients was reached ${ }^{[4]}$.

In the more contemporary disease-specific graded prognostic assessment (DS-GPA), the prognostic factors for patients with BM were attributed separately for respective primary tumors. For lung cancer, based on the results of 1833 NSCLC and 281 SCLC patients, apart from the prognostic factors from RTOG RPA class (extracranial disease, age and KPS) the number of BM (1 vs. 2-3 vs. > 3) reached prognostic significance. When patients with SCLC were analyzed separately, the number of BM was also significantly prognostic for survival ${ }^{[10]}$. The retrospective study demonstrated that median survival for 30 patients with single BM was 7 months compared with 2.9 months for 98 patients with multiple BM from SCLC $(P=0.005)^{[2]}$. In another small retrospective study, the number of BM also had an impact on survival ${ }^{[1]}$. The prognostic significance of the number of BM for SCLC may be counterintuitive, because we still treat this disease with WBRT based 
on the rationale that due to rapid progression, the subclinical disease foci exist in the whole brain at the onset of macroscopic BM. On the other hand, published data indicate that also in the case of SCLC, biological behavior may differ in a single BM compared with multiple BM. Thus, a disease that occurs with single or oligo- brain metastases may have different, more favorable prognosis than poly-metastatic brain disease at its onset. The latter would have greater inherent aggressiveness. However, we cannot exclude that in the case of less advanced brain involvement, more aggressive treatment strategies are employed. In the retrospective analysis of 52 patients who received WBRT for single BM, the use of surgery in combination with WBRT was related to improved survival compared with WBRT alone, with median overall survival of 19 and 5 months, respectively $(P=0.03)^{[12]}$. In the largest retrospective study on patients with BM from SCLC that included 229 patients treated with WBRT, the number of $\mathrm{BM}$ did not retain prognostic significance. Apart from the factors included in the RTOG RPA score, the time of occurrence of BM in relation to the diagnosis of the primary (synchronous vs. metachronous BM) and initial response to chemotherapy had a significant impact on survival. Patients presenting with synchronous BM and initial good response to chemotherapy had improved overall survival compared with patients presenting with metachronous BM and non-/poor responders to initial chemotherapy ${ }^{[13]}$. The authors proposed a new BM from SCLC score (BMS-score), which included RTOG RPA class and synchronous vs. metachronous BM presentation. Initial response to chemotherapy was not included in the score, because the evaluation of the response to chemotherapy may not be practical and as a result may prevent the use of the score on a large scale ${ }^{[14]}$.

Concluding the discussion of the issue of prognostic factors in BM from SCLC, we should emphasize the unquestioned value of the RTOG RPA classes. The role of the number of BM should be reconfirmed, especially in the era of rapid expansion of local treatment of BM, also in SCLC. The timing of occurrence of $\mathrm{BM}$, that is, synchronous $v s$. metachronous presentation, and the initial response to chemotherapy require further evaluation.

\section{TREATMENT OF BM WITH SYNCHRONOUS PRESENTATION}

BM that occur synchronously with primary diagnosis of SCLC represent different clinical scenarios and require different management compared with $\mathrm{BM}$ that are diagnosed metachronously, that is, at the relapse of SCLC. We may distinguish four categories of this synchronous presentation that require different management.

\section{BM diagnosed during an initial staging of SCLC}

When asymptomatic BM are part of the dissemination of SCLC, either as a sole distant site or as one of many distant locations, treatment usually starts with chemotherapy. The efficacy of chemotherapy for BM was questioned in the past, because the brain was considered a pharmacological sanctuary due to the brainblood barrier $(\mathrm{BBB})$ that prevented drugs from penetration into the brain. However, tumor invasion likely disrupts the $\mathrm{BBB}$, because there are many clinical observations of the efficacy of chemotherapy in BM from SCLC. Pooled data from five studies reported a $66 \%$ response rate (RR) in 64 patients with synchronous $\mathrm{BM}^{[15]}$. However, there are also contrary data indicating that the RR in the brain is lower than the systemic RR; with $27 \%$ vs. $73 \%$ RR for brain and extracranial sites, respectively, in 24 asymptomatic patients with BM from SCLC who received cyclophosphamide, doxorubicine, and vincristine ${ }^{[16]}$. However, an aggressive and rapid disease course prompts the initiation of systemic therapy so as not to miss the opportunity to administer chemotherapy that may stop extracranial systemic and local disease progression that causes bothersome and/or life threatening symptoms.

The question remains, whether chemotherapy should be followed by WBRT in such patients, even if the use of chemotherapy results in a complete response in the brain. We have no prospective data on this point. However, one randomized trial compared the use of teniposide alone vs. teniposide + WBRT 30 Gy in 120 
patients with progression in the brain after or during first-line treatment. The data from this study showed that omission of WBRT in the treatment of BM led to a significantly shorter time to progression in the brain in patients managed without WBRT, with $8 \% v s .35 \%$ of patients free of progression in the brain at 6 months in those treated with and without WBRT, respectively. Overall survival was short ( 3.5 months $)$ in both groups ${ }^{[5]}$. The demonstrated risk of rapid progression in the brain without the use of WBRT suggests the benefit of combining chemotherapy with WBRT also in these patients, regardless of the chemotherapy response-with the awareness that no strong evidence for such an approach exists.

\section{Histology of SCLC found during a craniotomy performed for brain tumor without prior diagnosis of lung primary with this histology}

This is a special case when the unexpected SCLC histology of a removed brain tumor leads to performing diagnostic procedures to find a lung primary and evaluate its extension. When the lung primary is found, further management does not differ from the scenario when BM are found during initial staging of SCLC, namely chemotherapy followed by WBRT. Patients with BM from SCLC were not included in the trials on the use of tumor bed radiosurgery with the omission of WBRT, because of the increased risk of dissemination outside the tumor bed ${ }^{[17,18]}$. Thus, also in these cases, WBRT remains the standard of care. A special presentation of BM SCLC represents a situation in which after removal of the brain tumor neither primary, nor signs of extracranial extension are found despite meticulous diagnostic procedures including PET-CT and bronchoscopy. In such a scenario, the justification for the use of chemotherapy may be questioned again, we have very limited evidence. The omission of chemotherapy as first-line treatment in such patients with careful monitoring may be an option in more fragile patients.

\section{BM developed during first-line treatment for ES-SCLC when no baseline brain imaging was performed}

Brain imaging in asymptomatic patients with ES-SCLC is not systematically performed. In the EORTC trial which demonstrated a survival advantage with the use of PCI, brain imaging was not part of the standard staging procedures and only $19 \%$ of randomized patients had baseline brain CT or MRI ${ }^{[8,19]}$. Obviously, if in such patients during first-line chemotherapy symptomatic BM occur, WBRT is given and chemotherapy is continued or discontinued after completion of brain irradiation depending on the systemic chemotherapy response and performance status of the patient.

\section{BM diagnosed before PCI for LS SCLC or ES SCLC in the case of pre-PCI MRI}

Brain imaging has not always been a standard procedure before PCI qualification in either LS SCLC or ES SCLC. Some prospective studies reported using CT scans, some did not require any imaging, and some did not mention any requirements for imaging use ${ }^{[20]}$. NCCN guidelines recommend pre-PCI MRI for patients with response to initial therapy ${ }^{[21]}$. These guidelines are strictly followed in the USA; as highlighted in a recent survey, up to $96 \%$ out of 309 US radiation oncologists performed pre-PCI MRI ${ }^{[22]}$. In contrast, some European IASLC and ESTRO experts indicated in a survey on the practice of PCI use for ES SCLC that the restrictions in reimbursement for MRI and problems with its availability were the main reasons of performing PCI in such patients. With MRI surveillance, patients would avoid brain irradiation, unnecessary in some cases $^{[23]}$. In one study, patients with initial diagnosis of LS SCLC had baseline MRI performed. Complete responders who qualified for PCI after treatment completion had a second, pre-PCI MRI; 13 out of 40 (32.5\%) patients had BM in pre-PCI MRI. Patients with pre-PCI detected BM had worse prognosis than those without BM in pre-PCI MRI ( $17 \%$ vs. $74 \%$ for 1-year survival, respectively, $P=0.0001)$. This difference was seen despite the higher and more intense WBRT doses in patients with BM. However, one major limitation of this study was that PCI was applied late, that is, 4-10 months after diagnosis. Moreover, this finding does not support the routine use of pre-PCI MRI, because even giving higher WBRT doses did not reverse the poor prognosis in these patients ${ }^{[24]}$. Similar observations from a larger group of patients were presented at the IASLC World Conference on Lung Cancer in 2018. From 119 LS SCLC patients referred for PCI after 
definitive chemoradiotherapy with baseline brain MRI, 25 (21\%) harbored BM on pre-PCI MRI, and 23 were asymptomatic. The duration of chemo-radiotherapy was the only prognostic factor for occurrence of pre-PCI BM. Patients with BM in pre-PCI MRI and duration of chemo-radiotherapy that exceeded 4.5 months had significantly shorter survival ${ }^{[25]}$. These findings are in line with modeling radiobiological studies that sought to elucidate dose-response relationship for PCI in SCLC as a function of time interval between time of treatment of primary tumor and initiation of PCI. The nearly linear dose-response relationship for reduction in BM was demonstrated for "early PCI" (up to 60 days from the start of treatment of the primary tumor) in doses of 0-35 Gy given in 2-Gy fractions. When PCI was delayed for over 60 days, a significant threshold in dose-response was observed, which is consistent with a fast growth rate of untreated subclinical BM from SCLC $^{[26]}$. Thus, it is important to initiate PCI early from the start of treatment. However, the guidelines do not recommend the concomitant use of PCI with chemotherapy because of apprehension concerning the toxicity of such an approach. What we learn from these studies is that every effort should be made to avoid unnecessarily prolonging chemo-radiotherapy for SCLC and to start PCI as quickly as possible after the end of treatment of the primary tumor. Pre-PCI MRI and higher WBRT dose not change a prognosis of asymptomatic patients in which progression in the brain occurs during initial therapy. However, in some patients with ES SCLC without BM on pre-PCI MRI, MRI surveillance without baseline PCI may be recommended as in a Japanese trial, in which patients with MRI surveillance did not have a survival benefit with the addition of PCI ${ }^{[27]}$. Pre-PCI MRI is also a pre-requisite for PCI with hippocampal avoidance.

\section{TREATMENT OF BM WITH METACHRONOUS PRESENTATION}

Despite a high initial response rate on chemotherapy, the majority of patients with LS SCLC and practically all with ES SCLC will relapse within the first year after treatment. After first-line chemotherapy, only extremely limited therapeutic options exist. Median survival after second-line chemotherapy is 3-6 months in clinical $\operatorname{trials}^{[28]}$. In addition, BM occur rarely as a sole event. The majority of patients with BM simultaneously have extracranial disease progression ${ }^{[4]}$. The limited therapeutic options and poor survival after relapse determine the poor outcome of treatment of metachronous BM. Thus, a metachronous presentation, that is, occurrence after first-line treatment, appears as an adverse prognostic factor ${ }^{[13,14]}$. Treatment strategies for BM that occur at the relapse differ with regard to the earlier use or not of PCI.

\section{Radiotherapy for BM that occur without prior PCI}

When the use of chemotherapy for asymptomatic BM that occur simultaneously with the diagnosis of primary is not debatable, the use of chemotherapy for metachronous BM is conditioned by a number of factors, such as the site of progression, brain only vs. brain and extracranial site, previous response to chemotherapy, the time interval from the last line of chemotherapy, the extent of extracranial disease, and performance status. We have no strong evidence for the use of chemotherapy for BM from SCLC. In the Cochrane Database of Systematic Reviews, only three small randomized trials involving 192 participants that dealt with chemotherapy for BM from SCLC were identified ${ }^{[29]}$. In one study, 120 patients with BM and concurrent systemic failure were randomized to receive teniposide with and without WBRT. Patients in the combined modality arm had higher RR in the brain (57\%) than patients treated with teniposide alone $(22 \%),(P<0.001)$. Patients who received WBRT also had longer time to progression in the brain than patients treated with chemotherapy alone, $(P=0.005)$. Overall survival did not differ greatly between the groups (median survival: 3.5 months in the combined modality arm and 3.2 months for chemotherapy alone arm; $P=0.087)^{[5]}$. Only one trial compared chemotherapy with no chemotherapy; 33 patients received WBRT for BM from SCLC (first line, $n=5$; recurrence, $n=28$ ) and were randomized to WBRT alone $v s$. WBRT plus topotecan. No significant difference in survival was found between these two groups ${ }^{[30]}$. The Cochrane Database of Systematic Reviews identified one other chemotherapy trial that compared two schedules - sequential and concomitant-of combination chemotherapy (teniposide plus cisplatin) with WBRT. This trial included only 39 patients and no difference in overall survival and response rate for either combination was demonstrated, although patients 
in the concomitant arm had a higher rate of myelosuppression ${ }^{[31]}$. Thus, the available evidence is insufficient to determine the effectiveness of chemotherapy for the treatment of BM from SCLC. We have no evidence that chemotherapy improves brain tumor control and overall survival in those patients. However, when BM are accompanied by systemic progression and the option of giving chemotherapy for a specific patient exists, chemotherapy is given based on the recognized chemo-responsiveness of this SCLC.

Although WBRT remains the standard therapeutic strategy for these patients, this approach is not based on the results of randomized trials. In addition, the effectiveness of WBRT in patients with widespread metastatic disease is debatable. We cannot exclude that some of these patients may be treated with chemotherapy alone. Another dubious indication for WBRT in these patients involves BM in RTOG RPA class 3, that is, patients with poor performance status (KPS $<70$ ). In one retrospective analysis of 132 patients with BM from SCLC who received WBRT, the median survival for 27 (20\%) patients from RPA class 3 was 2 months ${ }^{[2]}$; similarly, in another trial that included 154 such patients, the median survival for 51 (33\%) patients from RPA class 3 was 2.3 months $^{[3]}$. With such a short survival, the benefit of WBRT is doubtful. One prospective trial that also included patients with SCLC histology aimed to determine whether WBRT had any benefit in terms of symptoms palliation in 91 RTOG RPA class 3 patients. All patients received WBRT and were asked to complete a questionnaire about their symptoms before and 1 month after WBRT. Only 43 (47\%) patients completed the questionnaire after WBRT, the other patients died or were not able to respond to the survey questions because of further deterioration of performance and/or neurological status. In the group of patients who completed both questionnaires, the intensity of symptoms of the disease significantly increased after WBRT. This result challenges the value of WBRT for patients with poor performance status ${ }^{[32]}$. Recently, the limited value of WBRT in patients with BM from NSCLC who were unsuitable for resection or radiosurgery in terms of survival, quality of life, and reduction of the dose of steroids was demonstrated in a large randomized trial ${ }^{[33]}$. Taking into account, the short survival of patients with BM from SCLC, the value of considering WBRT in these patients as a standard of care should be subject to further research.

\section{Radiotherapy for BM that occur after PCI}

PCI reduces the risk of BM in both LS SCLC and ES SCLC, as shown in a meta-analysis and prospective randomized trials $s^{[6,8,27]}$. Nevertheless, a substantial proportion of patients still develop brain failure after PCI. The 3-year BM rate after PCI was 33\% vs. 59\% without PCI in a meta-analysis of 987 patients from seven randomized trials that compared treatment of SCLC with and without PCI ${ }^{[6]}$. In ES SCLC, the risk of symptomatic BM after PCI was $15 \%$ vs. $40 \%$ without $\mathrm{PCI}^{[8]}$. In a Japanese prospective trial that compared treatment with and without PCI with staging and strict surveillance with brain MRI, the 1-year BM rate was as high as $33 \%$ with PCI vs. $59 \%$ in patients without $\mathrm{PCI}^{[27]}$. These results indicate that $15 \%-30 \%$ of patients will still develop BM after PCI. Such failure represents a special therapeutic challenge, because reirradiation of the whole brain that has already received a biological dose of $\sim 30$ Gy risks meaningful neurotoxicity. On the other hand, the short survival of such patients may prevent them from the occurrence of late toxic effects, and in the short term, reirradiation at moderate doses may be beneficial, especially in symptomatic patients, taking into account the radio-sensitivity of SCLC. Even if localized treatment options like radiosurgery or less often resection appear as appropriate strategies in the irradiated region, most of these patients recur as multifocal BM that are unsuitable for radiosurgery. At the 2018 IASLC World Conference on Lung Cancer, one study reported that $60 \%$ of 32 patients who recurred in the brain after PCI were unsuitable for radiosurgery and WBRT may be considered for such patients ${ }^{[34]}$. There are very few reports about whole brain reirradiation after PCI. The largest series of patients treated with brain radiation after PCI with a median time of 14 months (range, 4-42 months) demonstrated that in 76 patients receiving a PCI dose of 30 Gy in 15 fractions, repeat WBRT with doses of 20-30 Gy in fractional doses of 2 Gy was given to $66(88 \%)$ patients and radiosurgery of $18-24$ Gy in a single fraction to $13(18 \%)$ patients. Median survival after repeat WBRT and radiosurgery was 3 months and 5 months, respectively, with a range of 0-12 months for both treatment types; $40 \%$ of 44 symptomatic patients improved after reirradiation. No serious, grade $>2$ toxicity was observed in these patients ${ }^{[35]}$. These results indicate that WBRT with moderate doses may be beneficial in 
Table 1. Characteristics and outcome of patients undergoing radiosurgery (RS) for brain metastases (BM) from small-cell lung cancer (SCLC)

\begin{tabular}{|c|c|c|c|c|c|c|}
\hline Author, year & $\begin{array}{l}\text { Number of } \\
\text { included } \\
\text { patients }\end{array}$ & $\begin{array}{c}\text { Number of BM } \\
\text { treated/doses of } \\
\text { SRS }\end{array}$ & $\begin{array}{l}\text { Number of patients } \\
\text { undergoing prior WBRT } \\
\text { (details on previous } \\
\text { treatment) }\end{array}$ & $\begin{array}{l}\text { Median overall } \\
\text { survival from } \\
\text { RS (months) }\end{array}$ & Local control & $\begin{array}{l}\text { Distant } \\
\text { control in the } \\
\text { brain }\end{array}$ \\
\hline Bernhardt et al. ${ }^{[35]}, 2016$ & 13 & $\begin{array}{l}\text { Maximum: } 4 \text { / 18- } \\
24 \text { Gy }\end{array}$ & 13 (PCl: 30 Gy in 15 fractions) & $\begin{array}{l}5 \text { (range: } \\
0-12 \text { ) }\end{array}$ & $\begin{array}{l}\text { Not } \\
\text { provided }\end{array}$ & Not provided \\
\hline Rava et al. ${ }^{[37]}, 2015$ & 40 & $\begin{array}{l}\text { Single: } 15 \\
2-3: 15 \\
\geq 4: 10 / \\
\text { No details }\end{array}$ & 37 (27: WBRT, 10: PCI) & $\begin{array}{l}6.5 \text { (range: } \\
4.1-8.9 \text { ) }\end{array}$ & $\begin{array}{l}\text { Actuarial } \\
\text { 1-year: 69\% }\end{array}$ & $\begin{array}{l}\text { Actuarial } \\
\text { 1-year: } 22 \%\end{array}$ \\
\hline Harris et al. ${ }^{[36]}, 2012$ & 51 & $\begin{array}{l}\text { Single: } 22 \\
\text { 2-3: } 18 \\
\geq 4: 11 / 10-24 \text { Gy } \\
\text { (median: } 18 \text { Gy) }\end{array}$ & 51 (35: WBRT, 16: PCI) & 5.9 & $\begin{array}{l}\text { Actuarial } \\
\text { 1-year: } 57 \%\end{array}$ & $\begin{array}{l}\text { Actuarial } \\
\text { 1-year: } 42 \%\end{array}$ \\
\hline Wegner et al. ${ }^{[40]}, 2011$ & 44 & $\begin{array}{l}\text { Median: } 1 \text { (range: } \\
\text { 1-14)/14-20 Gy } \\
\text { (median: } 18 \mathrm{Gy)}\end{array}$ & $\begin{array}{l}36 \text { (18: WBRT at median dose: } \\
30 \text { Gy in } 10 \text { fractions; } 6 \text { WBRT } \\
\text { combined with SRS; } 9: \text { PCI, 3: } \\
\text { PCI + WBRT for relapse) }\end{array}$ & 9 & $\begin{array}{l}\text { Actuarial } \\
\text { 1-year: } 86 \%\end{array}$ & $\begin{array}{l}\text { Crude rate of } \\
\text { failure: } 61 \%\end{array}$ \\
\hline Olson et al. $.^{[39]}, 2012$ & 27 & $\begin{array}{l}\text { Median: } 2 \\
\text { (range:1-6)/15-24 } \\
\text { Gy (median: } 20.5 \\
\text { Gy) }\end{array}$ & 27 (19: WBRT, 8: PCI) & 3 & $\begin{array}{l}\text { Actuarial } \\
\text { 1-year: } 75 \%\end{array}$ & $\begin{array}{l}\text { Actuarial } \\
\text { 1-year: } 31 \%\end{array}$ \\
\hline Yomo et al. $.^{[41]}, 2015$ & 70 & $\begin{array}{l}\text { Median: } 2 \\
\text { (range:1-21)/12-22 } \\
\text { Gy (median: 20 Gy) }\end{array}$ & $\begin{array}{l}23(16: \text { WBRT, } 7: \mathrm{PCI})+1 \\
\text { Hypofractionated partial } \\
\text { brain irradiation }\end{array}$ & $\begin{array}{l}7.8 \text { (range: } \\
0.6-56 \text { ) }\end{array}$ & $\begin{array}{l}\text { Actuarial } \\
\text { 1-year: 77\% }\end{array}$ & $\begin{array}{l}\text { Actuarial } \\
\text { 1-year: } 53 \%\end{array}$ \\
\hline Nakazaki et al. ${ }^{[38]}, 2012$ & 44 & $\begin{array}{l}\text { Median: } 5 \\
\text { (range:1-98)/10-21 } \\
\text { Gy (median: 20 Gy) }\end{array}$ & $\begin{array}{l}44 \text { (34: WBRT with median } \\
\text { dose of } 30 \mathrm{~Gy}, 10: \mathrm{PCI} \text { ) }\end{array}$ & $\begin{array}{l}5.8 \text { (range: } \\
0.5-24 \text { ) }\end{array}$ & $\begin{array}{l}33 \text { out of } 44 \\
\text { evaluated: } \\
\text { in } 10 \text { out of } \\
33 \text { failure: } \\
70 \% \text { crude } \\
\text { local control }\end{array}$ & $\begin{array}{l}33 \text { out of } 44 \\
\text { evaluated: in } \\
24 \text { out of } 33 \\
\text { failure: } 28 \% \\
\text { crude distant } \\
\text { control }\end{array}$ \\
\hline
\end{tabular}

Abbreviations: PCl: Prophylactic Cranial Irradiation; WBRT: Whole-Brain Radiotherapy

patients who are not candidates for radiosurgery and the short survival of such patients prevents them from the development of serious late neurotoxicity.

In the case of a limited number of $\mathrm{BM}<3 \mathrm{~cm}$ in diameter, the minimal invasiveness and ease of use of radiosurgery make it the preferred salvage method after prior PCI for patients with life expectancy $>3$ months. Retrospective data indicate that for patients with good performance status, radiosurgery for BM in SCLC gave results that were at least comparable with WBRT in terms of survival, with the median range of 3-9 months ${ }^{[30-36]}$. However, some reports reported lower local control after radiosurgery for BM from SCLC than for BM from other solid tumors. One-year local control rates were $<70 \%$ in evaluated patients ${ }^{[36-41]}$, whilst in prospective trials on radiosurgery with the exclusion of SCLC histology, these rates were $70 \%-90 \%{ }^{[42,43]}$. Distant brain control was also at the lower limit or $<60 \%$ as reported in prospective trials on radiosurgery alone ${ }^{[36-41]}$. This may be related to the known aggressiveness of SCLC, but also to the inclusion of patients with multiple ( $>4) \mathrm{BM}$, which may affect these results. Recently, it was demonstrated that radiosurgery without WBRT in patients with 5-10 $\mathrm{BM}$ was not inferior to that in patients with $2-4 \mathrm{BM}$ in terms of survival ${ }^{[4]}$. Table 1 summarizes the results of radiosurgery for BM from SCLC used as a salvage method after WBRT, as well as a first-line irradiation. To conclude, radiosurgery is an attractive treatment option after prior PCI and should be used if the technical possibilities for its use exist; however, WBRT at moderate doses is also feasible. WBRT is the treatment of first choice in patients who are unsuitable for radiosurgery or symptomatic patients with limited life expectancy $(<$ 3 months).

\section{REDUCTION OF NEUROTOXICITY IN BRAIN RADIOTHERAPY FOR SCLC}

WBRT in SCLC plays a role in prevention in the form of PCI and remains the standard in the treatment of BM. However, we have evidence that WBRT has a detrimental effect on neurocognitive functioning. In a 
phase III trial of standard-dose $v$ s. high-dose PCI for LS SCLC (RTOG0212), patients underwent evaluation for cognitive toxicity and quality of life effects. Patients receiving the higher-dose PCI were found to have a $25 \%$ increase in the rate of chronic cognitive toxicity compared with the standard-dose arm. However, $62 \%$ of patients receiving the standard-dose PCI also developed cognitive toxicity, as assessed by the Hopkins Verbal Learning Test (HVLT) Delayed Recall score ${ }^{[45]}$. These data indicate that even the standard-dose of PCI is associated with neurocognitive toxicity. It is also argued that with the improvement of survival, neurotoxicity has greater chance to occur and negatively impact quality of life $\mathrm{fe}^{[46]}$. Thus, the strategies to reduce neurotoxicity in PCI and WBRT warrant further investigation. One of these strategies is hippocampal avoidance (HA) during WBRT, based on the principle that proliferating neuronal progenitor cells in the subgranular zone of the hippocampus play an essential role in memory function. Thanks to technological advances and the availability of IMRT techniques, PCI and WBRT for BM with HA have been extensively explored and even used in routine practice. The RTOG 0933 trial demonstrated that HA during WBRT for BM was associated with a mean relative decline in the HVLT-Revised Delayed Recall score from baseline to 4 months of $7.0 \%$ (95\% confidence interval, $4.7 \%-18.7 \%$ ), which was a significant improvement compared with the historical control $(P=0.0003)^{[47]}$. Recently, for the first time, the benefit of HA in WBRT for BM in terms of preservation of neurocognitive function without compromising intracranial control was confirmed in 518 patients included in a phase III trial (NRG Oncology CC001). The 6-month neurocognitive function failure rate was $69 \%$ after WBRT without HA compared with $58 \%$ when using $\mathrm{HA}^{[48]}$.

However, the safety of this approach for SCLC remains to be demonstrated, because the risk of impaired intracranial control via underdosing some parts of the brain in the HA area persists in this very aggressive malignancy. Some reports support the safety of such an approach, indicating the risk of failure in the HA zone to be $<5 \%{ }^{[25,49]}$. In contrast, other reports warn of the rapid implementation of HA in SCLC, with an increased risk of failure of $>10 \%$ in the HA zone ${ }^{[50,51]}$. These contradictory data suggest that a potential small benefit of hippocampal sparing in limiting the neuropsychological sequelae of brain radiation may be obtained at the risk of failure in the spared region. In modeling studies, HA PCI in SCLC was more costeffective than conventional PCI provided that the risk of developing BM was not increased by more than $14 \%$, or if neurocognitive dysfunction rates were reduced by at least $40 \%{ }^{[52]}$. These findings strongly support the continued enrollment in ongoing cooperative group randomized trials on the value of HA PCI in SCLC: NRG CC003 (USA), PREMER (Spain) ${ }^{[53]}$, NCT01780675 (Holland).

Other strategy to reduce neurocognitive deterioration in patients after brain irradiation is the use of neuroprotective medication. The only agent that seems be effective in this indication is memantine. It was shown in the prospective study, that it delayed cognitive deterioration after WBRT, although at 24 weeks this did not reach significance $(P=0.059)^{[54]}$. Recently performed systematic review concluded that studies on other medications, like methylphenidate and donepezil remain inconclusive in this regard ${ }^{[5]}$.

\section{CONCLUSION}

To conclude, a standard radiation method for BM from SCLC remains WBRT. Stereotactic radiosurgery is reserved for relapses after PCI or prior WBRT for overt BM. However, the advancement in technologies allow for treatment of multiple metastases with omission of WBRT. Safety and benefit of such an approach remain to be confirmed in a prospective trial. All such efforts are welcomed. The ENCEPHALON trial launched at Heidelberg University Hospital randomizes patients with up to 10 metastases from SCLC to radiosurgery of all lesions $v s$. WBRT. Primary endpoint of the study is neurocognitive function. Secondary endpoints are intracranial control, overall survival and toxicity ${ }^{[56]}$. Only such direct comparisons will give evidence for safety of omission of WBRT in BM from SCLC. Mature data from the trials evaluating safety of HA in SCLC are also eagerly awaited. Short survival of such patients with both WBRT and stereotactic methods shows also that radiotherapy is not a sufficient treatment method in this indication. Combinations 
of radiotherapy with chemotherapy should be further evaluated. It was demonstrated in a randomized trial that Atezolizumb added to standard chemotherapy with Carboplatin and Etoposide in ES SCLC improved overall survival, however patients with BM represented only $8.5 \%$ of the included cohort ${ }^{[57]}$. Thus, further research is needed to investigate the role of immunotherapy in patients with BM from SCLC.

\section{DECLARATIONS}

\section{Authors' contributions}

The author solely contributed to the manuscript.

\section{Availability of data and materials}

Not applicable.

\section{Financial support and sponsorship}

None.

\section{Conflicts of interest}

All authors declared that there are no conflicts of interest.

\section{Ethical approval and consent to participate}

Not applicable.

\section{Consent for publication}

Not applicable.

\section{Copyright}

(c) The Author(s) 2019.

\section{REFERENCES}

1. Postmus PE. Brain metastases from small cell lung cancer: chemotherapy, radiotherapy, or both? Semin Radiat Oncol 1995;5:69-73.

2. Kepka L, Cieslak E, Bujko K. Wierzchowski M. Results of the whole-brain radiotherapy for patients with brain metastases from lung cancer: the RTOG RPA intra-classes analysis. Acta Oncol 2005;44:389-98.

3. Videtic GM, Adelstein DJ, Mekhail TM, Rice TW, Stevens GH, et al. Validation of the RTOG recursive partitioning analysis (RPA) classification for small-cell lung cancer-only brain metastases. Int J Radiat Oncol Biol Phys 2007;67:240-3.

4. Postmus PE, Haaxma-Reiche H, Gregor A, Groen HJ, Lewinski T, et al. Brain-only metastases of small cell lung cancer; efficacy of whole brain radiotherapy. An EORTC phase II study. Radiother Oncol 1998;46:29-32.

5. Postmus PE, Haaxma-Reiche H, Smit EF, Groen HJ, Karnicka H, et al. Treatment of brain metastases of small-cell lung cancer: comparing teniposide and teniposide with whole-brain radiotherapy--a phase III study of the European Organization for the Research and Treatment of Cancer Lung Cancer Cooperative Group. J Clin Oncol 2000;18:3400-8.

6. Auperin A, Arriagada R, Pignon JP, Le Péchoux C, Gregor A, et al. Prophylactic cranial irradiation for patients with small cell lung cancer in complete remission. Prophylactic Cranial Irradiation Overview Collaborative Group. N Engl J Med 1999;341:476-84.

7. Le Péchoux C, Dunant A, Senan S, Wolfson A, Quoix E, et al. Standard-dose versus higher-dose prophylactic cranial irradiation (PCI) in patients with limited-stage small-cell lung cancer in complete remission after chemotherapy and thoracic radiotherapy (PCI 99-01, EORTC 22003-08004, RTOG 0212, and IFCT 99-01): a randomised clinical trial. Lancet Oncol 2009;10:467-74.

8. Slotman B, Faivre-Finn C, Kramer G, Rankin E, Snee M, et al. Prophylactic cranial irradiation in extensive small-cell lung cancer. N Engl J Med 2007;357:664-72.

9. Gaspar L, Scott C, Rotman M, Asbell S, Phillips T, et al. Recursive partitioning analysis (RPA) of prognostic factors in three Radiation Therapy Oncology Group (RTOG) brain metastases trials. Int J Radiat Oncol Biol Phys 1997;37:745-51.

10. Sperduto PW, Kased N, Roberge D, Xu Z, Shanley R, et al. Summary report on the graded prognostic assessment: an accurate and facile diagnosis-specific tool to estimate survival for patients with brain metastases. J Clin Oncol 2012;30:419-25.

11. Rades D, Dziggel L, Segedin B, Oblak I, Nagy V, et al. The first survival score for patients with brain metastases from small cell lung cancer (SCLC). Clin Neurol Neurosurg 2013;115:2029-32.

12. Bernhardt D, Adeberg S, Bozorgmehr F, Opfermann N, Hörner-Rieber J, et al. Outcome and prognostic factors in single brain metastases 
from small-cell lung cancer. Strahlenther Oncol 2018;194:98-106.

13. Bernhardt D, Adeberg S, Bozorgmehr F, et al. Outcome and prognostic factors in patients with brain metastases from small-cell lung cancer treated with whole brain radiotherapy. J Neurooncol 2017;134:205-12.

14. Bernhardt D, König L, Aufderstrasse S, Krisam J, Hoerner-Rieber J, et al. Generation of a new disease-specific prognostic score for patients with brain metastases from small-cell lung cancer treated with whole brain radiotherapy (BMS-Score) and validation of two other indices. Clin Lung Cancer 2018;19:340-5.

15. Grossi F, Scolaro T, Tixi L, Loprevite M, Ardizzoni A. The role of systemic chemotherapy in the treatment of brain metastases from small-cell lung cancer. Crit Rev Oncol Hematol 2001;37:61-7.

16. Seute T, Leffers P, Wilmink JT, ten Velde GP, Twijnstra A. Response of asymptomatic brain metastases from small-cell lung cancer to systemic first-line chemotherapy. J Clin Oncol 2006;24:2079-83.

17. Brown PD, Ballman KV, Cerhan JH, Anderson SK, Carrero XW, et al. Postoperative stereotactic radiosurgery compared with whole brain radiotherapy for resected metastatic brain disease (NCCTG N107C/CEC.3): a multicentre, randomised, controlled, phase 3 trial. Lancet Oncol 2017;18:1049-60.

18. Kepka L, Tyc-Szczepaniak, Bujko K, Olszyna-Serementa M, Michalski W, et al. Stereotactic radiotherapy of the tumor bed compared to whole brain radiotherapy after surgery of single brain metastasis: results from a randomized trial. Radiother Oncol 2016;121:217-24.

19. Slotman B, Faivre-Finn C, Kramer G, Postmus B, Senan S, Prophylactic cranial irradiation in small-cell lung cancer. N Engl J Med 2007;357:1978.

20. Davey P, Ennis M, Aviv R. Prophylactic cranial irradiation (PCI). Still a no-brainer? Lung Cancer 2015;89:4-7.

21. National Comprehensive Cancer Network Clinical Practice Guidelines in Oncology. Small Cell Lung Cancer (Version 1.2019).

22. Farell MJ, Yahya JB, Degnin C, Chen Y, Holland JM, et al. Prophylactic cranial irradiation for limited-stage small cell lung cancer: A survey of US radiation oncologists on current practice patterns. Clin Lung Cancer 2019;20:13-9.

23. Putora PM, Glatzer M, Belderbos J, Besse B, Blackhall F, et al. Prophylactic cranial irradiation in stage IV small cell lung cancer: Selection of patients amongst European IASLC and ESTRO experts. Radiother Oncol 2019;133;163-6.

24. Manapov F, Klautke G, Fietkau R. Prevalence of brain metastases immediately before prophylactic cranial irradiation in limited disease small cell lung cancer patients with complete remission to chemoradiotherapy: a single institution experience. J Thoracic Oncol 2008;3:652-5.

25. Chu X, Yang X, Zhu Z. Prevalence and risk factors of brain metastases in limited stage small cell lung cancer immediately before prophylactic cranial irradiation. J Thorac Oncol 2018;10:S436.

26. Suwinski R, Lee SP, Withers HR. Dose-response relationship for prophylactic cranial irradiation in small cell lung cancer. Int J Radiat Oncol Biol Phys 1998;40:797-806.

27. Takahashi T, Yamanaka T, Seto T, Harada H, Nokihara H, et al. Prophylactic cranial irradiation versus observation in patients with extensive-disease small-cell lung cancer: a multicentre, randomised, open-label, phase 3 trial. Lancet Oncol 2017;18:663-71.

28. Puglisi M, Dolly S, Faria A, Myerson JS, Popat S, et al. Treatment options for small cell lung cancer - do we have more choice? Br J Cancer 2010;102:629-38.

29. Reveiz L, Rueda JR, Cardona AF. Chemotherapy for brain metastases from small cell lung cancer. Cochrane Database Syst Rev 2012;6:CD007464.

30. Neuhaus T, Ko Y, Muller RP, Grabenbauer GG, Hedde JP, et al. A phase III trial of topotecan and whole brain radiation therapy for patients with CNS-metastases due to lung cancer. Br J Cancer 2009;100:291-7.

31. Liu M, Zhou Y, Han Q, Gao T, Luo Z, Wang W. Whole brain radiotherapy concomitant or sequential Vm26/DDP in treating small cell lung cancer patients with brain metastases. Chinese-German Journal of Clinical Oncology 2010;9:17-21.

32. Komosinska K, Kepka L, Niwinska A, Pietrzak L, Wierzchowski M, et al. Prospective evaluation of the palliative effect of whole-brain radiotherapy in patients with brain metastases and poor performance status. Acta Oncol 2010;49:382-8.

33. Mulvenna P, Nankivell M, Barton R, Faivre-Finn C, Wilson P, et al. Dexamethasone and supportive care with or without whole brain radiotherapy in treating patients with non-small cell lung cancer with brain metastases unsuitable for resection or stereotactic radiotherapy (QUARTZ): results from a phase 3, non-inferiority, randomised trial. Lancet 2016;388:2004-14.

34. Mazure B, Guest N, Letcher A, et al. Should stereotactic radiosurgery be considered for salvage of intracranial recurrence in small cell lung cancer? J Thoracic Oncol 2018;13:S436.

35. Bernhardt D, Bozorgmehr F, Adeberg S, Opfermann N, von Eiff D, et al. Outcome in patients with small cell lung cancer re-irradiated for brain metastases after prior prophylactic cranial irradiation. Lung Cancer 2016;101:76-81.

36. Harris S, Chan MD, Lovato JF, Ellis TL, Tatter SB, et al. Gamma knife stereotactic radiosurgery as salvage therapy after failure of whole-brain radiotherapy in patients with small cell lung cancer. Int J Radiat Oncol Biol Phys 2012;83:e53-9.

37. Rava P, Sioshansi S, DiPetrillo T, Cosgrove R, Melhus C, et al. Local recurrence and survival following stereotactic radiosurgery for brain metastases from small cell lung cancer. Pract Radiat Oncol 2015;5:e37-44.

38. Nakazaki K, Higuchi Y, Nagano O, and Serizawa T. Efficacy and limitations of salvage gamma knife radiosurgery for brain metastases of small-cell lung cancer after whole-brain radiotherapy. Acta Neurochir 2013;155:107-14.

39. Olson AC, Wegner RE, Rwigema JC, Heron DE, Burton SA, et al. Clinical outcomes of reirradiation of brain metastases from small cell lung cancer with cyberknife stereotactic radiosurgery. J Cancer Res Ther 2012;8:411-6.

40. Wegner RE, Olson AC, Kondziolka D, Niranjan A, Lundsford LD, et al. Stereotactic radiosurgery for patients with brain metastases from small cell lung cancer. Int J Radiat Oncol Biol Phys 2011;81:e21-7.

41. Yomo S, Hayashi M. Is stereotactic radiosurgery a rational treatment option for brain metastases from small cell lung cancer? A retrospective analysis of 70 consecutive patients. BMC Cancer 2015;15:95. 
42. Aoyama H, Shirato H, Tago M, Nakagawa K, Toyoda T, et al. Stereotactic radiosurgery plus whole-brain radiation therapy vs stereotactic radiosurgery alone for treatment of brain metastases: a randomized controlled trial. JAMA 2006;295:2483-91.

43. Kocher M, Soffietti R, Abacioglu U, Villà S, Fauchon F, et al. Adjuvant whole-brain radiotherapy versus observation after radiosurgery or surgical resection of one to three cerebral metastases: results of the EORTC 22952-26001 study. J Clin Oncol 2011;29:134-41.

44. Yamamoto M, Serizawa T, Shuto T, Akabane A, Higuchi Y, et al. Stereotactic radiosurgery for patients with multiple brain metastases (JLGK0901): a multi-institutional prospective observational study. Lancet Oncol 2014;15:387-95.

45. Wolfson AH, Bae K, Komaki R, Meyers C, Movsas B, et al. Primary analysis of a phase II randomized trial Radiation Therapy Oncology Group (RTOG) 0212: impact of different total doses and schedules of prophylactic cranial irradiation on chronic neurotoxicity and quality of life for patients with limited-disease small-cell lung cancer. Int J Radiat Oncol Biol Phys 2011;81:77-84.

46. Socha J, Kepka L. Prophylactic cranial irradiation for small-cell lung cancer: how, when and for whom? Expert Rev Anticancer Ther 2012;12:505-17.

47. Gondi V, Pugh SL, Tome WA, Caine C, Corn B, et al. Preservation of memory with conformal avoidance of the hippocampal neural stem-cell compartment during whole-brain radiotherapy for brain metastases (RTOG0933): a phase II multi-institutional trial. J Clin Oncol 2014;32:3810-6.

48. Gondi V, Deshmukh S, Brown PD, et al. Preservation of neurocognitive function (NCF) with conformal avoidance of the hippocampus during whole-brain radiotherapy (HA-WBRT) for brain metastases: preliminary results of phase III trial NRG Oncology CC001. Int J Radiat Oncol Biol Phys 2018;102:1607S.

49. Kundapur V, Ellchuk T, Ahmed S, Gondi V. Risk of hippocampal metastases in small cell lung cancer patients at presentation and after cranial irradiation: a safety profile study for hippocampal sparing during prophylactic or therapeutic cranial irradiation. Int J Radiat Oncol Biol Phys 2015;91:781-6.

50. Harth S, Abo-Madyan Y, Zheng L, Siebenlist K, Herskind C, et al. Estimation of intracranial failure risk following hippocampal-sparing whole brain radiotherapy. Radiother Oncol 2013;109:152-8.

51. Redmond KJ, Hales RK, Anderson-Keightly H, Zhou XC, Kummerlowe M, et al. Prospective study of hippocampal-sparing prophylactic cranial irradiation in limited-stage small cell lung cancer. Int J Radiat Oncol Biol Phys 2017;98:603-11.

52. Qu XM, Mishra MV, Bauman GS, Slotman B, Mehta M, et al. Cost-effectiveness of prophylactic cranial irradiation with hippocampal avoidance in limited stage small cell lung cancer. Radiother Oncol 2017;122:411-5.

53. Rodriguez de Dios N, Counago F, Lopez JL, Calvo P, Murcia M, et al. Treatment design and rationale for a randomized trial of prophylactic cranial irradiation with or without hippocampal avoidance for sclc: premer trial on behalf of the oncologic group for the study of lung cancer/spanish radiation oncology group-radiation oncology clinical research group. Clin Lung Cancer 2018;19:e693-7.

54. Brown PD, Pugh S, Laack NN, Wefel JS, Khuntia D, et al. Memantine for the prevention of cognitive dysfunction in patients receiving whole-brain radiotherapy: a randomized, double-blind, placebo-controlled trial. Neuro Oncol. 2013;15:1429-37.

55. Wartena R, Brandsma D, Belderbos J. Are memantine, methylphenidate and donepezil effective in sparing cognitive functioning after brain irradiation? J Cancer Metastasis Treat 2018;4:59.

56. Bernhardt D, Hommertgen A, Schmitt D, El Shafie R, Paul A, et al. Whole brain radiation therapy alone versus radiosurgery for patients with 1-10 brain metastases from small cell lung cancer (ENCEPHALON Trial): study protocol for a randomized controlled trial. Trials 2018;19:388.

57. Horn L, Mansfield AS, Szczesna A, Havel L, Krzakowski M, et al. First-line Atezolizumab plus chemotherapy in Extensive-Stage Small-Cell Lung Cancer. N Engl J Med 2018;379:2220-9. 